\title{
INEQUALITIES OF HERMITE-HADAMARD TYPE FOR COMPOSITE $h$-CONVEX FUNCTIONS
}

\author{
SILVESTRU SEVER DRAGOMIR ${ }^{1,2}$
}

\begin{abstract}
In this paper we obtain some inequalities of Hermite-Hadamard type for composite convex functions. Applications for $A G, A H$ - $h$-convex functions, $G A, G G, G H$-h-convex functions and $H A, H G, H H$ - $h$-convex function are given.
\end{abstract}

\section{INTRODUCTION}

We recall here some concepts of convexity that are well known in the literature. Let $I$ be an interval in $\mathbb{R}$.

Definition 1 ([52]). We say that $f: I \rightarrow \mathbb{R}$ is a Godunova-Levin function or that $f$ belongs to the class $Q(I)$ if $f$ is non-negative and for all $x, y \in I$ and $t \in(0,1)$ we have

$$
f(t x+(1-t) y) \leq \frac{1}{t} f(x)+\frac{1}{1-t} f(y) .
$$

Some further properties of this class of functions can be found in [42], [43], [45], [58], [64] and [65]. Among others, its has been noted that non-negative monotone and non-negative convex functions belong to this class of functions.

Definition $2([45])$. We say that a function $f: I \rightarrow \mathbb{R}$ belongs to the class $P(I)$ if it is nonnegative and for all $x, y \in I$ and $t \in[0,1]$ we have

$$
f(t x+(1-t) y) \leq f(x)+f(y) .
$$

Obviously $Q(I)$ contains $P(I)$ and for applications it is important to note that also $P(I)$ contain all nonnegative monotone, convex and quasi convex functions, i. e. nonnegative functions satisfying

$$
f(t x+(1-t) y) \leq \max \{f(x), f(y)\}
$$

for all $x, y \in I$ and $t \in[0,1]$.

For some results on $P$-functions see [45] and [62] while for quasi convex functions, the reader can consult [44].

Definition $3([10])$. Let $s$ be a real number, $s \in(0,1]$. A function $f:[0, \infty) \rightarrow$ $[0, \infty)$ is said to be s-convex (in the second sense) or Breckner s-convex if

$$
f(t x+(1-t) y) \leq t^{s} f(x)+(1-t)^{s} f(y)
$$

for all $x, y \in[0, \infty)$ and $t \in[0,1]$.

1991 Mathematics Subject Classification. 26D15; 26D10.

Key words and phrases. Convex functions, $A G, A H$ - $h$-convex functions, $G A, G G, G H-h-$ convex functions and $H A, H G, H H$-h-convex function, Integral inequalities. 
For some properties of this class of functions see [2], [3], [10], [11], [40], [41], [53], [55] and [67].

In order to unify the above concepts for functions of real variable, S. Varošanec introduced the concept of $h$-convex functions as follows.

Assume that $I$ and $J$ are intervals in $\mathbb{R},(0,1) \subseteq J$ and functions $h$ and $f$ are real non-negative functions defined in $J$ and $I$, respectively.

Definition 4 ([70]). Let $h: J \rightarrow[0, \infty)$ with $h$ not identical to 0 . We say that $f: I \rightarrow[0, \infty)$ is an h-convex function if for all $x, y \in I$ we have

$$
f(t x+(1-t) y) \leq h(t) f(x)+h(1-t) f(y)
$$

for all $t \in(0,1)$.

For some results concerning this class of functions see [70], [9], [56], [68], [66] and [69].

We can introduce now another class of functions.

Definition 5. We say that the function $f: I \rightarrow[0, \infty)$ is of $s$-Godunova-Levin type, with $s \in[0,1]$, if

$$
f(t x+(1-t) y) \leq \frac{1}{t^{s}} f(x)+\frac{1}{(1-t)^{s}} f(y),
$$

for all $t \in(0,1)$ and $x, y \in I$.

We observe that for $s=0$ we obtain the class of $P$-functions while for $s=1$ we obtain the class of Godunova-Levin. If we denote by $Q_{s}(C)$ the class of $s$ Godunova-Levin functions defined on $C$, then we obviously have

$$
P(C)=Q_{0}(C) \subseteq Q_{s_{1}}(C) \subseteq Q_{s_{2}}(C) \subseteq Q_{1}(C)=Q(C)
$$

for $0 \leq s_{1} \leq s_{2} \leq 1$.

If $f: I \rightarrow[0, \infty)$ is an $h$-convex function on an interval $I$ of real numbers with $h \in L[0,1]$ and $f \in L[a, b]$ with $a, b \in I, a<b$, then we have the Hermite-Hadamard type inequality obtained by Sarikaya et al. in [66]

$$
\begin{aligned}
\frac{1}{2 h\left(\frac{1}{2}\right)} f\left(\frac{a+b}{2}\right) & \leq \frac{1}{b-a} \int_{a}^{b} f(u) d u \\
& =\int_{0}^{1} f((1-\lambda) a+\lambda b) d \lambda \leq[f(a)+f(b)] \int_{0}^{1} h(t) d t
\end{aligned}
$$

For an extension of this result to functions defined on convex subsets of linear spaces and refinements, see [31].

In order to extend this result for other classes of functions, we need the following preparations.

Let $g:[a, b] \rightarrow[g(a), g(b)]$ be a continuous strictly increasing function that is differentiable on $(a, b)$.

Definition 6. A function $f:[a, b] \rightarrow \mathbb{R}$ will be called composite- $g^{-1} h$-convex (concave) on $[a, b]$ if the composite function $f \circ g^{-1}:[g(a), g(b)] \rightarrow \mathbb{R}$ is $h$-convex (concave) in the usual sense on $[g(a), g(b)]$.

If $f:[a, b] \rightarrow \mathbb{R}$ is composite- $g^{-1} h$-convex on $[a, b]$ then we have the inequality

$$
f \circ g^{-1}((1-\lambda) u+\lambda v) \leq h(1-\lambda) f \circ g^{-1}(u)+h(\lambda) f \circ g^{-1}(v)
$$

for any $u, v \in[g(a), g(b)]$ and $\lambda \in[0,1]$. 
This is equivalent to the condition

$$
f \circ g^{-1}((1-\lambda) g(t)+\lambda g(s)) \leq h(1-\lambda) f(t)+h(\lambda) f(s)
$$

for any $t, s \in[a, b]$ and $\lambda \in[0,1]$.

If we take $g(t)=\ln t, t \in[a, b] \subset(0, \infty)$, then the condition (1.8) becomes

$$
f\left(t^{1-\lambda} s^{\lambda}\right) \leq h(1-\lambda) f(t)+h(\lambda) f(s)
$$

for any $t, s \in[a, b]$ and $\lambda \in[0,1]$, which is the concept of $G A$-h-convexity as considered in [1].

If we take $g(t)=-\frac{1}{t}, t \in[a, b] \subset(0, \infty)$, then (1.8) becomes

$$
f\left(\frac{t s}{(1-\lambda) s+\lambda t}\right) \leq h(1-\lambda) f(t)+h(\lambda) f(s)
$$

for any $t, s \in[a, b]$ and $\lambda \in[0,1]$, which is the concept of $H A$-h-convexity as considered in [5].

If $p>0$ and we consider $g(t)=t^{p}, t \in[a, b] \subset(0, \infty)$, then the condition (1.8) becomes

$$
f\left[\left((1-\lambda) t^{p}+\lambda s^{p}\right)^{1 / p}\right] \leq h(1-\lambda) f(t)+h(\lambda) f(s)
$$

for any $t, s \in[a, b]$ and $\lambda \in[0,1]$. For $h(t)=t$ the concept of $p$-convexity was considered in [71].

If we take $g(t)=\exp t, t \in[a, b]$, then the condition (1.8) becomes

$$
f[\ln ((1-\lambda) \exp (t)+\exp g(s))] \leq(1-\lambda) f(t)+\lambda f(s),
$$

which is the concept of LogExp h-convex function on $[a, b]$. For $h(t)=t$, the concept was considered in [28].

Further, assume that $f:[a, b] \rightarrow \mathcal{J}, \mathcal{J}$ an interval of real numbers and $k: \mathcal{J} \rightarrow \mathbb{R}$ a continuous function on $\mathcal{J}$ that is strictly increasing (decreasing) on $\mathcal{J}$.

Definition 7. We say that the function $f:[a, b] \rightarrow \mathcal{J}$ is $k$-composite $h$-convex (concave) on $[a, b]$, if $k \circ f$ is h-convex (concave) on $[a, b]$.

With $g:[a, b] \rightarrow[g(a), g(b)]$ a continuous strictly increasing function that is differentiable on $(a, b), f:[a, b] \rightarrow \mathcal{J}, \mathcal{J}$ an interval of real numbers and $k: \mathcal{J} \rightarrow \mathbb{R}$ a continuous function on $\mathcal{J}$ that is strictly increasing (decreasing) on $\mathcal{J}$, we can also consider the following concept:

Definition 8. We say that the function $f:[a, b] \rightarrow \mathcal{J}$ is $k$-composite- $g^{-1} h$-convex (concave) on $[a, b]$, if $k \circ f \circ g^{-1}$ is h-convex (concave) on $[g(a), g(b)]$.

This definition is equivalent to the condition

$$
k \circ f \circ g^{-1}((1-\lambda) g(t)+\lambda g(s)) \leq h(1-\lambda)(k \circ f)(t)+h(\lambda)(k \circ f)(s)
$$

for any $t, s \in[a, b]$ and $\lambda \in[0,1]$.

If $k: \mathcal{J} \rightarrow \mathbb{R}$ is strictly increasing (decreasing) on $\mathcal{J}$, then the condition (1.13) is equivalent to:

$$
\begin{aligned}
f \circ g^{-1}((1-\lambda) g(t)+ & \lambda g(s)) \\
\leq & (\geq) k^{-1}[h(1-\lambda)(k \circ f)(t)+h(\lambda)(k \circ f)(s)]
\end{aligned}
$$

for any $t, s \in[a, b]$ and $\lambda \in[0,1]$. 
If $k(t)=\ln t, t>0$ and $f:[a, b] \rightarrow(0, \infty)$, then the fact that $f$ is $k$-composite $h$-convex on $[a, b]$ is equivalent to the fact that $f$ is log-convex or multiplicatively convex or $A G$-h-convex, namely, for all $x, y \in I$ and $t \in[0,1]$ one has the inequality:

$$
f(t x+(1-t) y) \leq[f(x)]^{h(t)}[f(y)]^{h(1-t)} .
$$

A function $f: I \rightarrow \mathbb{R} \backslash\{0\}$ is called $A H$-h-convex (concave) on the interval $I$ if the following inequality holds [1]

$$
f((1-\lambda) x+\lambda y) \leq(\geq) \frac{f(x) f(y)}{h(1-\lambda) f(y)+h(\lambda) f(x)}
$$

for any $x, y \in I$ and $\lambda \in[0,1]$.

An important case that provides many examples is that one in which the function is assumed to be positive for any $x \in I$. In that situation the inequality (1.16) is equivalent to

$$
h(1-\lambda) \frac{1}{f(x)}+h(\lambda) \frac{1}{f(y)} \leq(\geq) \frac{1}{f((1-\lambda) x+\lambda y)}
$$

for any $x, y \in I$ and $\lambda \in[0,1]$.

Taking into account this fact, we can conclude that the function $f: I \rightarrow(0, \infty)$ is $A H$ - $h$-convex (concave) on $I$ if and only if $f$ is $k$-composite $h$-concave (convex) on $I$ with $k:(0, \infty) \rightarrow(0, \infty), k(t)=\frac{1}{t}$.

Following [1], we can introduce the concept of $G H$-h-convex (concave) function $f: I \subset(0, \infty) \rightarrow \mathbb{R}$ on an interval of positive numbers $I$ as satisfying the condition

$$
f\left(x^{1-\lambda} y^{\lambda}\right) \leq(\geq) \frac{f(x) f(y)}{h(1-\lambda) f(y)+h(\lambda) f(x)} .
$$

Since

$$
f\left(x^{1-\lambda} y^{\lambda}\right)=f \circ \exp [(1-\lambda) \ln x+\lambda \ln y]
$$

and

$$
\frac{f(x) f(y)}{h(1-\lambda) f(y)+h(\lambda) f(x)}=\frac{f \circ \exp (\ln x) f \circ \exp (\ln y)}{h(1-\lambda) f \circ \exp (y)+h(\lambda) f \circ \exp (x)}
$$

then $f: I \subset(0, \infty) \rightarrow \mathbb{R}$ is $G H$-convex (concave) on $I$ if and only if $f \circ \exp$ is $A H$ convex (concave) on $\ln I:=\{x \mid x=\ln t, t \in I\}$. This is equivalent to the fact that $f$ is $k$-composite- $g^{-1} h$-concave (convex) on $I$ with $k:(0, \infty) \rightarrow(0, \infty), k(t)=\frac{1}{t}$ and $g(t)=\ln t, t \in I$. if

Following [1], we say that the function $f: I \subset \mathbb{R} \backslash\{0\} \rightarrow(0, \infty)$ is $H H$-h-convex

$$
f\left(\frac{x y}{t x+(1-t) y}\right) \leq \frac{f(x) f(y)}{h(1-t) f(y)+h(t) f(x)}
$$

for all $x, y \in I$ and $t \in[0,1]$. If the inequality in (1.18) is reversed, then $f$ is said to be $H H$-h-concave.

We observe that the inequality (1.18) is equivalent to

$$
h(1-t) \frac{1}{f(x)}+h(t) \frac{1}{f(y)} \leq \frac{1}{f\left(\frac{x y}{t x+(1-t) y}\right)}
$$

for all $x, y \in I$ and $t \in[0,1]$.

This is equivalent to the fact that $f$ is $k$-composite- $g^{-1} h$-concave on $[a, b]$ with $k:(0, \infty) \rightarrow(0, \infty), k(t)=\frac{1}{t}$ and $g(t)=-\frac{1}{t}, t \in[a, b]$. 
The function $f: I \subset(0, \infty) \rightarrow(0, \infty)$ is called $G G$-h-convex on the interval $I$ of real umbers $\mathbb{R}$ if $[5]$

$$
f\left(x^{1-\lambda} y^{\lambda}\right) \leq[f(x)]^{h(1-\lambda)}[f(y)]^{h(\lambda)}
$$

for any $x, y \in I$ and $\lambda \in[0,1]$. If the inequality is reversed in (1.20) then the function is called $G G-h$-concave.

For $h(t)=t$, this concept was introduced in 1928 by P. Montel [59], however, the roots of the research in this area can be traced long before him [60]. It is easy to see that [60], the function $f:[a, b] \subset(0, \infty) \rightarrow(0, \infty)$ is $G G$-h-convex if and only if the the function $g:[\ln a, \ln b] \rightarrow \mathbb{R}, g=\ln \circ f \circ \exp$ is $h$-convex on $[\ln a, \ln b]$. This is equivalent to the fact that $f$ is $k$-composite- $g^{-1} h$-convex on $[a, b]$ with $k:(0, \infty) \rightarrow \mathbb{R}, k(t)=\ln t$ and $g(t)=\ln t, t \in[a, b]$. if

Following [1] we say that the function $f: I \subset \mathbb{R} \backslash\{0\} \rightarrow(0, \infty)$ is $H G$-h-convex

$$
f\left(\frac{x y}{t x+(1-t) y}\right) \leq[f(x)]^{h(1-t)}[f(y)]^{h(t)}
$$

for all $x, y \in I$ and $t \in[0,1]$. If the inequality in (1.8) is reversed, then $f$ is said to be $H G$-h-concave.

Let $f:[a, b] \subset(0, \infty) \rightarrow(0, \infty)$ and define the associated functions $G_{f}:\left[\frac{1}{b}, \frac{1}{a}\right] \rightarrow$ $\mathbb{R}$ defined by $G_{f}(t)=\ln f\left(\frac{1}{t}\right)$. Then $f$ is $H G$ - $h$-convex on $[a, b]$ iff $G_{f}$ is $h$-convex on $\left[\frac{1}{b}, \frac{1}{a}\right]$. This is equivalent to the fact that $f$ is $k$-composite- $g^{-1} h$-convex on $[a, b]$ with $k:(0, \infty) \rightarrow \mathbb{R}, k(t)=\ln t$ and $g(t)=-\frac{1}{t}, t \in[a, b]$.

We say that the function $f:[a, b] \rightarrow(0, \infty)$ is $r$-h-convex, for $r \neq 0$, if

$$
f((1-\lambda) x+\lambda y) \leq\left[h(1-\lambda) f^{r}(y)+h(\lambda) f^{r}(x)\right]^{1 / r}
$$

for any $x, y \in[a, b]$ and $\lambda \in[0,1]$. For $h(t)=t$, the concept was considered in [61],

If $r>0$, then the condition (1.22) is equivalent to

$$
f^{r}((1-\lambda) x+\lambda y) \leq h(1-\lambda) f^{r}(y)+h(\lambda) f^{r}(x)
$$

namely $f$ is $k$-composite convex on $[a, b]$ where $k(t)=t^{r}, t \geq 0$.

If $r<0$, then the condition (1.22) is equivalent to

$$
f^{r}((1-\lambda) x+\lambda y) \geq h(1-\lambda) f^{r}(y)+h(\lambda) f^{r}(x)
$$

namely $f$ is $k$-composite $h$-concave on $[a, b]$ where $k(t)=t^{r}, t>0$.

In this paper we obtain some inequalities of Hermite-Hadamard type for composite convex functions. Applications for $A G, A H$ - $h$-convex functions, $G A, G G$, $G H$-h-convex functions and $H A, H G, H H$-h-convex function are given.

\section{Refinements of $H H$-Inequality}

The following representation result holds.

Lemma 1. Let $f: I \subseteq \mathbb{R} \rightarrow \mathbb{C}$ where $I$ is an interval of the real numbers $\mathbb{R}$. Let $y, x \in I$ with $y \neq x$ and assume that the mapping $[0,1] \ni t \mapsto f \circ$ $g^{-1}[(1-t) g(x)+t g(y)]$ is Lebesgue integrable on $[0,1]$. Then for any $\lambda \in[0,1]$ 
we have the representation

$$
\begin{aligned}
& \int_{0}^{1} f \circ g^{-1}[(1-t) g(x)+t g(y)] d t \\
& =(1-\lambda) \int_{0}^{1} f \circ g^{-1}[(1-t)((1-\lambda) g(x)+\lambda g(y))+t g(y)] d t \\
& \quad+\lambda \int_{0}^{1} f \circ g^{-1}[(1-t) g(x)+t((1-\lambda) g(x)+\lambda g(y))] d t .
\end{aligned}
$$

Proof. For $\lambda=0$ and $\lambda=1$ the equality (2.1) is obvious.

Let $\lambda \in(0,1)$. Observe that

$$
\begin{aligned}
& \int_{0}^{1} f \circ g^{-1}[(1-t)(\lambda g(y)+(1-\lambda) g(x))+t g(y)] d t \\
& =\int_{0}^{1} f \circ g^{-1}[((1-t) \lambda+t) g(y)+(1-t)(1-\lambda) g(x)] d t
\end{aligned}
$$

and

$$
\begin{aligned}
& \int_{0}^{1} f \circ g^{-1}[t(\lambda g(y)+(1-\lambda) g(x))+(1-t) g(x)] d t \\
& =\int_{0}^{1} f \circ g^{-1}[t \lambda g(y)+(1-\lambda t) g(x)] d t .
\end{aligned}
$$

If we make the change of variable $u:=(1-t) \lambda+t$ then we have $1-u=$ $(1-t)(1-\lambda)$ and $d u=(1-\lambda) d u$. Then

$$
\begin{aligned}
& \int_{0}^{1} f \circ g^{-1}[((1-t) \lambda+t) g(y)+(1-t)(1-\lambda) g(x)] d t \\
& =\frac{1}{1-\lambda} \int_{\lambda}^{1} f \circ g^{-1}[u g(y)+(1-u) g(x)] d u .
\end{aligned}
$$

If we make the change of variable $u:=\lambda t$ then we have $d u=\lambda d t$ and

$$
\int_{0}^{1} f \circ g^{-1}[t \lambda g(y)+(1-\lambda t) g(x)] d t=\frac{1}{\lambda} \int_{0}^{\lambda} f \circ g^{-1}[u g(y)+(1-u) g(x)] d u \text {. }
$$

Therefore

$$
\begin{aligned}
& (1-\lambda) \int_{0}^{1} f \circ g^{-1}[(1-t)(\lambda g(y)+(1-\lambda) g(x))+t g(y)] d t \\
& +\lambda \int_{0}^{1} f \circ g^{-1}[t(\lambda g(y)+(1-\lambda) g(x))+(1-t) g(x)] d t \\
& =\int_{\lambda}^{1} f \circ g^{-1}[u g(y)+(1-u) g(x)] d u+\int_{0}^{\lambda} f \circ g^{-1}[u g(y)+(1-u) g(x)] d u \\
& =\int_{0}^{1} f \circ g^{-1}[u g(y)+(1-u) g(x)] d u
\end{aligned}
$$

and the identity (2.1) is proved. 
Theorem 1. Assume that the function $f: I \subseteq \mathbb{R} \rightarrow[0, \infty)$ is a composite- $g^{-1}$ $h$-convex function with $h \in L[0,1]$. Let $y, x \in I$ with $y \neq x$, then for any $\lambda \in[0,1]$ we have the inequalities

$$
\begin{aligned}
& \frac{1}{2 h\left(\frac{1}{2}\right)}\left\{(1-\lambda) f \circ g^{-1}\left[\frac{(1-\lambda) g(x)+(\lambda+1) g(y)}{2}\right]\right. \\
& \left.+\lambda f \circ g^{-1}\left[\frac{(2-\lambda) g(x)+\lambda g(y)}{2}\right]\right\} \\
& \leq \frac{1}{g(y)-g(x)} \int_{x}^{y} f(t) g^{\prime}(t) d t \\
& \leq\left[f \circ g^{-1}((1-\lambda) g(x)+\lambda g(y))+(1-\lambda) f(y)+\lambda f(x)\right] \int_{0}^{1} h(t) d t \\
& \leq\{[h(1-\lambda)+\lambda] f(x)+[h(\lambda)+1-\lambda] f(y)\} \int_{0}^{1} h(t) d t .
\end{aligned}
$$

If $f: I \subseteq \mathbb{R} \rightarrow[0, \infty)$ is a composite- $g^{-1} h$-concave function, then the inequalities reverse in (2.2).

Proof. Since $f: I \subseteq \mathbb{R} \rightarrow[0, \infty)$ is a composite- $g^{-1} h$-convex function function, then by Hermite-Hadamard type inequality (1.6) we have

$$
\begin{aligned}
& \frac{1}{2 h\left(\frac{1}{2}\right)} f \circ g^{-1}\left[\frac{(1-\lambda) g(x)+(\lambda+1) g(y)}{2}\right] \\
& \leq \int_{0}^{1} f \circ g^{-1}[(1-t)((1-\lambda) g(x)+\lambda g(y))+t g(y)] d t \\
& \leq\left[f \circ g^{-1}((1-\lambda) g(x)+\lambda g(y))+f(y)\right] \int_{0}^{1} h(t) d t
\end{aligned}
$$

and

$$
\begin{aligned}
& \frac{1}{2 h\left(\frac{1}{2}\right)} f \circ g^{-1}\left[\frac{(2-\lambda) g(x)+\lambda g(y)}{2}\right] \\
& \leq \int_{0}^{1} f \circ g^{-1}[(1-t) g(x)+t((1-\lambda) g(x)+\lambda g(y))] d t \\
& \leq\left[f(x)+f \circ g^{-1}((1-\lambda) g(x)+\lambda g(y))\right] \int_{0}^{1} h(t) d t .
\end{aligned}
$$

Now, if we multiply the inequality (2.3) by $1-\lambda \geq 0$ and (2.4) by $\lambda \geq 0$ and add the obtained inequalities, then we get

$$
\begin{aligned}
& \frac{1-\lambda}{2 h\left(\frac{1}{2}\right)} f \circ g^{-1}\left[\frac{(1-\lambda) g(x)+(\lambda+1) g(y)}{2}\right] \\
& +\frac{\lambda}{2 h\left(\frac{1}{2}\right)} f \circ g^{-1}\left[\frac{(2-\lambda) g(x)+\lambda g(y)}{2}\right]
\end{aligned}
$$




$$
\begin{aligned}
& \leq(1-\lambda) \int_{0}^{1} f \circ g^{-1}[(1-t)((1-\lambda) g(x)+\lambda g(y))+t g(y)] d t \\
& +\lambda \int_{0}^{1} f \circ g^{-1}[(1-t) g(x)+t((1-\lambda) g(x)+\lambda g(y))] d t \\
& \leq(1-\lambda)\left[f \circ g^{-1}((1-\lambda) g(x)+\lambda g(y))+f(y)\right] \int_{0}^{1} h(t) d t \\
& +\lambda\left[f(x)+f \circ g^{-1}((1-\lambda) g(x)+\lambda g(y))\right] \int_{0}^{1} h(t) d t
\end{aligned}
$$

and by (2.1) we obtain

$$
\begin{aligned}
& \frac{1}{2 h\left(\frac{1}{2}\right)}\left\{(1-\lambda) f \circ g^{-1}\left[\frac{(1-\lambda) g(x)+(\lambda+1) g(y)}{2}\right]\right. \\
& \left.+\lambda f \circ g^{-1}\left[\frac{(2-\lambda) g(x)+\lambda g(y)}{2}\right]\right\} \\
& \leq \int_{0}^{1} f \circ g^{-1}[(1-t) g(x)+t g(y)] d t \\
& \leq\left[f \circ g^{-1}((1-\lambda) g(x)+\lambda g(y))+(1-\lambda) f(y)+\lambda f(x)\right] \int_{0}^{1} h(t) d t \\
& \leq\{[h(1-\lambda)+\lambda] f(x)+[h(\lambda)+1-\lambda] f(y)\} \int_{0}^{1} h(t) d t,
\end{aligned}
$$

where the last inequality follows by the definition of composite- $g^{-1} h$-convexity and performing the required calculation.

By using the change of variable $u=(1-t) g(x)+t g(y)$, we have $d u=(g(y)-g(x)) d t$ and then

$$
\int_{0}^{1} f \circ g^{-1}[(1-t) g(x)+t g(y)] d t=\frac{1}{g(y)-g(x)} \int_{g(x)}^{g(y)} f \circ g^{-1}(u) d u .
$$

If we change the variable $t=g^{-1}(u)$, then $u=g(t)$, which gives that $d u=g^{\prime}(t) d t$ and then

$$
\int_{g(x)}^{g(y)} f \circ g^{-1}(u) d u=\int_{x}^{y} f(t) g^{\prime}(t) d t
$$

and the inequality (2.2) is obtained.

Remark 1. With the assumptions from Theorem 1, we observe that if we take either $\lambda=0$ or $\lambda=1$ in the first two inequalities in (2.2), then we get (1.6). 
If we take $\lambda=\frac{1}{2}$ and use the $h$-convexity of $f \circ g^{-1}$, then we get from (2.2) that

$$
\begin{aligned}
& \frac{1}{4 h^{2}\left(\frac{1}{2}\right)} f \circ g^{-1}\left(\frac{g(x)+g(y)}{2}\right) \\
& \leq \frac{1}{4 h\left(\frac{1}{2}\right)}\left\{f \circ g^{-1}\left(\frac{g(x)+3 g(y)}{4}\right)+f \circ g^{-1}\left(\frac{3 g(x)+g(y)}{4}\right)\right\} \\
& \leq \frac{1}{g(y)-g(x)} \int_{x}^{y} f(t) g^{\prime}(t) d t \\
& \leq\left[f \circ g^{-1}\left(\frac{g(x)+g(y)}{2}\right)+\frac{f(x)+f(y)}{2}\right] \int_{0}^{1} h(t) d t \\
& \leq\left[h\left(\frac{1}{2}\right)+\frac{1}{2}\right][f(x)+f(y)] \int_{0}^{1} h(t) d t,
\end{aligned}
$$

where $y, x \in I$ with $y \neq x$.

Remark 2. In general, if $h(\lambda)>0$ for $\lambda \in(0,1)$, then for $y, x \in I$ with $y \neq x$

$$
\begin{gathered}
(1-\lambda) f \circ g^{-1}\left[\frac{(1-\lambda) g(x)+(\lambda+1) g(y)}{2}\right] \\
+\lambda f \circ g^{-1}\left[\frac{(2-\lambda) g(x)+\lambda g(y)}{2}\right] \\
=\frac{1-\lambda}{h(1-\lambda)} h(1-\lambda) f \circ g^{-1}\left[\frac{(1-\lambda) g(x)+(\lambda+1) g(y)}{2}\right] \\
+\frac{\lambda}{h(\lambda)} h(\lambda) f \circ g^{-1}\left[\frac{(2-\lambda) g(x)+\lambda g(y)}{2}\right] \\
\geq \min \left\{\frac{1-\lambda}{h(1-\lambda)}, \frac{\lambda}{h(\lambda)}\right\} \\
\times\left\{h(1-\lambda) f \circ g^{-1}\left[\frac{(1-\lambda) g(x)+(\lambda+1) g(y)}{2}\right]\right. \\
\left.+h(\lambda) f \circ g^{-1}\left[\frac{(2-\lambda) g(x)+\lambda g(y)}{2}\right]\right\} \\
\geq \min \left\{\frac{1-\lambda}{h(1-\lambda)}, \frac{\lambda}{h(\lambda)}\right\} \\
\times f \circ g^{-1}\left[(1-\lambda) \frac{(1-\lambda) g(x)+(\lambda+1) g(y)}{2}+\lambda \frac{(2-\lambda) g(x)+\lambda g(y)}{2}\right] \\
=\min \left\{\frac{1-\lambda}{h(1-\lambda)}, \frac{\lambda}{h(\lambda)}\right\} f \circ g^{-1}\left(\frac{g(x)+g(y)}{2}\right)
\end{gathered}
$$

and from (2.2) we get the sequence of inequalities

$$
\begin{aligned}
\frac{1}{2 h\left(\frac{1}{2}\right)} \min \left\{\frac{1-\lambda}{h(1-\lambda)}, \frac{\lambda}{h(\lambda)}\right\} f \circ g^{-1}\left(\frac{g(x)+g(y)}{2}\right) \\
\leq \frac{1}{2 h\left(\frac{1}{2}\right)}\left\{(1-\lambda) f \circ g^{-1}\left[\frac{(1-\lambda) g(x)+(\lambda+1) g(y)}{2}\right]\right. \\
\left.+\lambda f \circ g^{-1}\left[\frac{(2-\lambda) g(x)+\lambda g(y)}{2}\right]\right\}
\end{aligned}
$$


10

$$
\begin{aligned}
& \leq \frac{1}{g(y)-g(x)} \int_{x}^{y} f(t) g^{\prime}(t) d t \\
& \quad \leq\left[f \circ g^{-1}((1-\lambda) g(x)+\lambda g(y))+(1-\lambda) f(y)+\lambda f(x)\right] \int_{0}^{1} h(t) d t \\
& \quad \leq\{[h(1-\lambda)+\lambda] f(x)+[h(\lambda)+1-\lambda] f(y)\} \int_{0}^{1} h(t) d t
\end{aligned}
$$

for $y, x \in I$ with $y \neq x$.

In particular, we have

$$
\begin{aligned}
& \frac{1}{4 h^{2}\left(\frac{1}{2}\right)} f \circ g^{-1}\left(\frac{g(x)+g(y)}{2}\right) \\
& \leq \frac{1}{4 h\left(\frac{1}{2}\right)}\left\{f \circ g^{-1}\left[\frac{(1-\lambda) g(x)+(\lambda+1) g(y)}{2}\right]\right. \\
& \left.+f \circ g^{-1}\left[\frac{(2-\lambda) g(x)+\lambda g(y)}{2}\right]\right\} \\
& \leq \frac{1}{g(y)-g(x)} \int_{x}^{y} f(t) g^{\prime}(t) d t \quad \leq\left[h\left(\frac{1}{2}\right)+\frac{1}{2}\right][f(x)+f(y)] \int_{0}^{1} h(t) d t .
\end{aligned}
$$

In a similar way, if $f: I \subseteq \mathbb{R} \rightarrow[0, \infty)$ is a composite- $g^{-1} h$-concave function, then

$$
\begin{array}{r}
\frac{1}{2 h\left(\frac{1}{2}\right)} \max \left\{\frac{1-\lambda}{h(1-\lambda)}, \frac{\lambda}{h(\lambda)}\right\} f \circ g^{-1}\left(\frac{g(x)+g(y)}{2}\right) \\
\geq \frac{1}{2 h\left(\frac{1}{2}\right)}\left\{(1-\lambda) f \circ g^{-1}\left[\frac{(1-\lambda) g(x)+(\lambda+1) g(y)}{2}\right]\right. \\
\left.+\lambda f \circ g^{-1}\left[\frac{(2-\lambda) g(x)+\lambda g(y)}{2}\right]\right\} \\
\geq \frac{1}{g(y)-g(x)} \int_{x}^{y} f(t) g^{\prime}(t) d t \\
\geq\left[f \circ g^{-1}((1-\lambda) g(x)+\lambda g(y))+(1-\lambda) f(y)+\lambda f(x)\right] \int_{0}^{1} h(t) d t \\
\geq\{[h(1-\lambda)+\lambda] f(x)+[h(\lambda)+1-\lambda] f(y)\} \int_{0}^{1} h(t) d t,
\end{array}
$$

for $y, x \in I$ with $y \neq x$. 
In particular,

$$
\begin{aligned}
& \frac{1}{4 h^{2}\left(\frac{1}{2}\right)} f \circ g^{-1}\left(\frac{g(x)+g(y)}{2}\right) \\
& \geq \frac{1}{4 h\left(\frac{1}{2}\right)}\left\{f \circ g^{-1}\left[\frac{(1-\lambda) g(x)+(\lambda+1) g(y)}{2}\right]\right. \\
& \left.+f \circ g^{-1}\left[\frac{(2-\lambda) g(x)+\lambda g(y)}{2}\right]\right\} \\
& \geq \frac{1}{g(y)-g(x)} \int_{x}^{y} f(t) g^{\prime}(t) d t \\
& \geq\left[f \circ g^{-1}\left(\frac{g(x)+g(y)}{2}\right)+\frac{f(y)+f(x)}{2}\right] \int_{0}^{1} h(t) d t \\
& \geq\left[h\left(\frac{1}{2}\right)+\frac{1}{2}\right][f(x)+f(y)] \int_{0}^{1} h(t) d t .
\end{aligned}
$$

Corollary 1. Let $f: I \subseteq \mathbb{R} \rightarrow[0, \infty)$ be a composite- $g^{-1}$ convex function on the interval $I$ in $\mathbb{R}$. Then for any $y, x \in I$ with $y \neq x$ and for any $\lambda \in[0,1]$ we have the inequalities

$$
\begin{gathered}
f \circ g^{-1}\left(\frac{g(x)+g(y)}{2}\right) \leq(1-\lambda) f \circ g^{-1}\left[\frac{(1-\lambda) g(x)+(\lambda+1) g(y)}{2}\right] \\
+\lambda f \circ g^{-1}\left[\frac{(2-\lambda) g(x)+\lambda g(y)}{2}\right] \\
\leq \frac{1}{g(y)-g(x)} \int_{x}^{y} f(t) g^{\prime}(t) d t \\
\leq \frac{1}{2}\left[f \circ g^{-1}((1-\lambda) g(x)+\lambda g(y))+(1-\lambda) f(y)+\lambda f(x)\right] \\
\leq \frac{f(y)+f(x)}{2} .
\end{gathered}
$$

We have:

Corollary 2. Let $f: I \subseteq \mathbb{R} \rightarrow[0, \infty)$ be a composite- $g^{-1}$ Breckner s-convex function on the interval $I$ with $s \in(0,1]$. Then for any $y, x \in I$ with $y \neq x$ and for any $\lambda \in[0,1]$ we have the inequalities

$$
\begin{aligned}
& \frac{1}{2^{1-s}}\left(\frac{1}{2}-\left|\lambda-\frac{1}{2}\right|\right)^{1-s} f \circ g^{-1}\left(\frac{g(x)+g(y)}{2}\right) \\
& \leq \frac{1}{2^{1-s}}\left\{(1-\lambda) f \circ g^{-1}\left[\frac{(1-\lambda) g(x)+(\lambda+1) g(y)}{2}\right]\right. \\
& \left.+\lambda f \circ g^{-1}\left[\frac{(2-\lambda) g(x)+\lambda g(y)}{2}\right]\right\}
\end{aligned}
$$




$$
\begin{aligned}
& \leq \frac{1}{g(y)-g(x)} \int_{x}^{y} f(t) g^{\prime}(t) d t \\
& \leq \frac{1}{s+1}\left[f \circ g^{-1}((1-\lambda) g(x)+\lambda g(y))+(1-\lambda) f(y)+\lambda f(x)\right] \\
& \quad \leq \frac{1}{s+1}\left\{\left[(1-\lambda)^{s}+\lambda\right] f(x)+\left(\lambda^{s}+1-\lambda\right) f(y)\right\}
\end{aligned}
$$

We also have:

Corollary 3. Let $f: I \subseteq \mathbb{R} \rightarrow[0, \infty)$ be a composite- $g^{-1}$ of $s$-Godunova-Levin type on the interval $I$ with $s \in(0,1)$. Then for any $y, x \in I$ with $y \neq x$ and for any $\lambda \in(0,1)$ we have the inequalities

$$
\begin{gathered}
\frac{1}{2^{1+s}}\left(\frac{1}{2}-\left|\lambda-\frac{1}{2}\right|\right)^{1+s} f \circ g^{-1}\left(\frac{g(x)+g(y)}{2}\right) \\
\leq \frac{1}{2^{1+s}}\left\{(1-\lambda) f \circ g^{-1}\left[\frac{(1-\lambda) g(x)+(\lambda+1) g(y)}{2}\right]\right. \\
\left.+\lambda f \circ g^{-1}\left[\frac{(2-\lambda) g(x)+\lambda g(y)}{2}\right]\right\} \\
\leq \frac{1}{g(y)-g(x)} \int_{x}^{y} f(t) g^{\prime}(t) d t \\
\leq \frac{1}{1-s}\left[f \circ g^{-1}((1-\lambda) g(x)+\lambda g(y))+(1-\lambda) f(y)+\lambda f(x)\right] \\
\leq \frac{1}{1-s}\left\{\left[(1-\lambda)^{-s}+\lambda\right] f(x)+\left(\lambda^{-s}+1-\lambda\right) f(y)\right\}
\end{gathered}
$$

More generally, we have:

Corollary 4. Assume that $g:[a, b] \rightarrow[g(a), g(b)]$ is a continuous strictly increasing function that is differentiable on $(a, b), f:[a, b] \rightarrow \mathcal{J}, \mathcal{J}$ an interval of real numbers and $k: \mathcal{J} \rightarrow \mathbb{R}$ is a continuous function on $\mathcal{J}$ that is strictly increasing. If the function $f:[a, b] \rightarrow \mathcal{J}$ is $k$-composite- $g^{-1} h$-convex on $[a, b]$, then

$$
\begin{aligned}
\frac{1}{2 h\left(\frac{1}{2}\right)} \min \left\{\frac{1-\lambda}{h(1-\lambda)}, \frac{\lambda}{h(\lambda)}\right\} & k \circ f \circ g^{-1}\left(\frac{g(x)+g(y)}{2}\right) \\
\leq \frac{1}{2 h\left(\frac{1}{2}\right)}\left\{(1-\lambda) k \circ f \circ g^{-1}\right. & {\left[\frac{(1-\lambda) g(x)+(\lambda+1) g(y)}{2}\right] } \\
& \left.+\lambda k \circ f \circ g^{-1}\left[\frac{(2-\lambda) g(x)+\lambda g(y)}{2}\right]\right\}
\end{aligned}
$$

$$
\begin{aligned}
& \leq \frac{1}{g(y)-g(x)} \int_{x}^{y}(k \circ f)(t) g^{\prime}(t) d t \\
& \leq\left[k \circ f \circ g^{-1}((1-\lambda) g(x)+\lambda g(y))+(1-\lambda)(k \circ f)(y)+\lambda(k \circ f)(x)\right] \\
& \quad \times \int_{0}^{1} h(t) d t \\
& \quad \leq\{[h(1-\lambda)+\lambda](k \circ f)(x)+[h(\lambda)+1-\lambda](k \circ f)(y)\} \int_{0}^{1} h(t) d t,
\end{aligned}
$$

for $y, x \in[a, b]$ with $y \neq x$. 
If the function $f:[a, b] \rightarrow \mathcal{J}$ is $k$-composite- $g^{-1} h$-concave on $[a, b]$, then

$$
\begin{array}{r}
\frac{1}{2 h\left(\frac{1}{2}\right)} \max \left\{\frac{1-\lambda}{h(1-\lambda)}, \frac{\lambda}{h(\lambda)}\right\} \\
\geq \frac{1}{2 h\left(\frac{1}{2}\right)}\left\{(1-\lambda) k \circ f \circ g^{-1}\left[\frac{(1-\lambda) g(x)+(\lambda+1) g(y)}{2}\right]\right. \\
\left.+\lambda k \circ f \circ g^{-1}\left[\frac{(2-\lambda) g(x)+\lambda g(y)}{2}\right]\right\} \\
\geq \frac{1}{g(y)-g(x)} \int_{x}^{y}(k \circ f)(t) g^{\prime}(t) d t \\
\geq\left[k \circ f \circ g^{-1}((1-\lambda) g(x)+\lambda g(y))+(1-\lambda)(k \circ f)(y)+\lambda(k \circ f)(x)\right] \\
\quad \times \int_{0}^{1} h(t) d t \\
\geq\{[h(1-\lambda)+\lambda](k \circ f)(x)+[h(\lambda)+1-\lambda](k \circ f)(y)\} \int_{0}^{1} h(t) d t,
\end{array}
$$

for $y, x \in[a, b]$ with $y \neq x$.

The proof follows by the inequalities (2.8) and (2.10) and we omit the details.

In 1906, Fejér [51], while studying trigonometric polynomials, obtained the following inequalities which generalize that of Hermite \& Hadamard:

Theorem 2 (Fejér's Inequality). Consider the integral $\int_{a}^{b} h(x) w(x) d x$, where $h$ is a convex function in the interval $(a, b)$ and $w$ is a positive function in the same interval such that

$$
w(x)=w(a+b-x), \text { for any } x \in[a, b]
$$

i.e., $y=w(x)$ is a symmetric curve with respect to the straight line which contains the point $\left(\frac{1}{2}(a+b), 0\right)$ and is normal to the $x$-axis. Under those conditions the following inequalities are valid:

$$
h\left(\frac{a+b}{2}\right) \int_{a}^{b} w(x) d x \leq \int_{a}^{b} h(x) w(x) d x \leq \frac{h(a)+h(b)}{2} \int_{a}^{b} w(x) d x .
$$

If $h$ is concave on $(a, b)$, then the inequalities reverse in (2.18).

If $w:[a, b] \rightarrow \mathbb{R}$ is continuous and positive on the interval $[a, b]$, then the function $W:[a, b] \rightarrow[0, \infty), W(x):=\int_{a}^{x} w(s) d s$ is strictly increasing and differentiable on $(a, b)$ and the inverse $W^{-1}:\left[a, \int_{a}^{b} w(s) d s\right] \rightarrow[a, b]$ exists.

Remark 3. Assume that $w:[a, b] \rightarrow \mathbb{R}$ is continuous and positive on the interval $[a, b], f:[a, b] \rightarrow \mathcal{J}, \mathcal{J}$ an interval of real numbers and $k: \mathcal{J} \rightarrow \mathbb{R}$ is a continuous 
function on $\mathcal{J}$ that is strictly increasing. If the function $f:[a, b] \rightarrow \mathcal{J}$ is $k$ composite- $W^{-1} h$-convex on $[a, b]$, then we have the weighted inequality

$$
\begin{gathered}
\text { 19) } \frac{1}{2 h\left(\frac{1}{2}\right)} \min \left\{\frac{1-\lambda}{h(1-\lambda)}, \frac{\lambda}{h(\lambda)}\right\} k \circ f \circ W^{-1}\left(\frac{\int_{a}^{x} w(s) d s+\int_{a}^{y} w(s) d s}{2}\right) \\
\leq \frac{1}{2 h\left(\frac{1}{2}\right)}\left\{(1-\lambda) k \circ f \circ W^{-1}\left[\frac{(1-\lambda) \int_{a}^{x} w(s) d s+(\lambda+1) \int_{a}^{y} w(s) d s}{2}\right]\right. \\
\left.+\lambda k \circ f \circ W^{-1}\left[\frac{\left.(2-\lambda) \int_{a}^{x} w(s) d s+\lambda \int_{a}^{y} w(s) d s\right]}{2}\right]\right\} \\
\leq \frac{1}{\int_{x}^{y} w(s) d s} \int_{x}^{y}(k \circ f)(t) w(t) d t \\
\leq\left[k \circ f \circ W^{-1}\left((1-\lambda) \int_{a}^{x} w(s) d s+\lambda \int_{a}^{y} w(s) d s\right)\right. \\
\quad+(1-\lambda)(k \circ f)(y)+\lambda(k \circ f)(x)] \int_{0}^{1} h(t) d t \\
\leq\{[h(1-\lambda)+\lambda](k \circ f)(x)+[h(\lambda)+1-\lambda](k \circ f)(y)\} \int_{0}^{1} h(t) d t,
\end{gathered}
$$

for any $\lambda \in[0,1]$ and for $y, x \in[a, b]$ with $y \neq x$.

\section{Applichtions for $A G$ and $A H$ - $h$-Convex Functions}

The function $f:[a, b] \rightarrow(0, \infty)$ is $A G$ - $h$-convex means that $f$ is $k$-composite $h$-convex on $[a, b]$ with $k(t)=\ln t, t>0$. By making use of Corollary 4 for $g(t)=t$, we get

$$
\begin{gathered}
{\left[f\left(\frac{x+y}{2}\right)\right]^{\frac{1}{2 h\left(\frac{1}{2}\right)} \min \left\{\frac{1-\lambda}{h(1-\lambda)}, \frac{\lambda}{h(\lambda)}\right\}}} \\
\leq\left\{f^{(1-\lambda)}\left[\frac{(1-\lambda) x+(\lambda+1) y}{2}\right] f^{\lambda}\left[\frac{(2-\lambda) x+\lambda y}{2}\right]\right\}^{\frac{1}{2 h\left(\frac{1}{2}\right)}} \\
\leq \exp \left(\frac{1}{y-x} \int_{x}^{y} \ln f(t) d t\right) \\
\leq\left[f((1-\lambda) x+\lambda y) f^{(1-\lambda)}(y) f^{\lambda}(x)\right]^{\int_{0}^{1} h(t) d t} \\
\leq\left\{f^{[h(1-\lambda)+\lambda]}(x) f^{[h(\lambda)+1-\lambda]}(y)\right\}^{\int_{0}^{1} h(t) d t},
\end{gathered}
$$

for any $\lambda \in[0,1]$ and $x, y \in[a, b]$ with $y \neq x$.

The function $f:[a, b] \rightarrow(0, \infty)$ is $A H$-h-convex on $[a, b]$ means that $f$ is $k$ composite $h$-concave on $[a, b]$ with $k:(0, \infty) \rightarrow(0, \infty), k(t)=\frac{1}{t}$. By making use 
of Corollary 4 for $g(t)=t$, we get

$$
\begin{aligned}
& \frac{1}{2 h\left(\frac{1}{2}\right)} \max \left\{\frac{1-\lambda}{h(1-\lambda)}, \frac{\lambda}{h(\lambda)}\right\} f^{-1}\left(\frac{x+y}{2}\right) \\
& \geq \frac{1}{2 h\left(\frac{1}{2}\right)}\left\{(1-\lambda) f^{-1}\left[\frac{(1-\lambda) x+(\lambda+1) y}{2}\right]\right. \\
& \left.+\lambda f^{-1}\left[\frac{(2-\lambda) x+\lambda y}{2}\right]\right\} \\
& \geq \frac{1}{y-x} \int_{x}^{y} f^{-1}(t) d t \\
& \geq\left[f^{-1}((1-\lambda) x+\lambda y)+(1-\lambda) f^{-1}(y)+\lambda f^{-1}(x)\right] \int_{0}^{1} h(t) d t \\
& \geq\left\{[h(1-\lambda)+\lambda] f^{-1}(x)+[h(\lambda)+1-\lambda] f^{-1}(y)\right\} \int_{0}^{1} h(t) d t,
\end{aligned}
$$

for any $\lambda \in[0,1]$ and $x, y \in[a, b]$ with $y \neq x$.

\section{Applications for $G A, G G$ and $G H$ - $h$-Convex Functions}

If we take $g(t)=\ln t, t \in[a, b] \subset(0, \infty)$, then $f:[a, b] \rightarrow \mathbb{R}$ is $G A$-h-convex on $[a, b]$ means that that $f:[a, b] \rightarrow \mathbb{R}$ composite- $g^{-1} h$-convex on $[a, b]$. By making use of Corollary 4 for $k(t)=t$, we get

$$
\begin{aligned}
& \frac{1}{2 h\left(\frac{1}{2}\right)} \min \left\{\frac{1-\lambda}{h(1-\lambda)}, \frac{\lambda}{h(\lambda)}\right\} f(\sqrt{x y}) \\
& \leq \frac{1}{2 h\left(\frac{1}{2}\right)}\left\{(1-\lambda) f\left(x^{\frac{1-\lambda}{2}} y^{\frac{\lambda+1}{2}}\right)+\lambda f\left(x^{\frac{2-\lambda}{2}} y^{\frac{\lambda}{2}}\right)\right\} \\
& \leq \frac{1}{\ln \left(\frac{y}{x}\right)} \int_{x}^{y} \frac{f(t)}{t} d t \\
& \leq\left[f\left(x^{1-\lambda} y^{\lambda}\right)+(1-\lambda) f(y)+\lambda f(x)\right] \int_{0}^{1} h(t) d t \\
& \leq\{[h(1-\lambda)+\lambda] f(x)+[h(\lambda)+1-\lambda] f(y)\} \int_{0}^{1} h(t) d t
\end{aligned}
$$

for any $\lambda \in[0,1]$ and for $y, x \in[a, b]$ with $y \neq x$.

The function $f: I \subset(0, \infty) \rightarrow(0, \infty)$ is $G G$ - $h$-convex means that $f$ is $k$ composite- $g^{-1} h$-convex on $[a, b]$ with $k:(0, \infty) \rightarrow \mathbb{R}, k(t)=\ln t$ and $g(t)=\ln t$, $t \in[a, b]$. By making use of Corollary 4 we get

$$
\begin{aligned}
& {[f(\sqrt{x y})]^{\frac{1}{2 h\left(\frac{1}{2}\right)} \min \left\{\frac{1-\lambda}{h(1-\lambda)}, \frac{\lambda}{h(\lambda)}\right\}}} \\
& \leq\left\{f^{(1-\lambda)}\left(x^{\frac{1-\lambda}{2}} y^{\frac{\lambda+1}{2}}\right) f^{\lambda}\left(x^{\frac{2-\lambda}{2}} y^{\frac{\lambda}{2}}\right)\right\}^{\frac{1}{2 h\left(\frac{1}{2}\right)}}
\end{aligned}
$$




$$
\begin{aligned}
& \leq \exp \left(\frac{1}{\ln \left(\frac{y}{x}\right)} \int_{x}^{y} \frac{\ln f(t)}{t} d t\right) \\
& \leq\left[f\left(x^{1-\lambda} y^{\lambda}\right) f^{\lambda}(x) f^{1-\lambda}(y)\right]^{\int_{0}^{1} h(t) d t} \\
& \leq\left\{f^{[h(1-\lambda)+\lambda]}(x) f^{[h(\lambda)+1-\lambda]}(y)\right\}^{\int_{0}^{1} h(t) d t},
\end{aligned}
$$

for any $\lambda \in[0,1]$ and for $y, x \in[a, b]$ with $y \neq x$.

We also have that $f:[a, b] \subset(0, \infty) \rightarrow \mathbb{R}$ is $G H$-h-convex on $[a, b]$ is equivalent to the fact that $f$ is $k$-composite- $g^{-1} h$-concave on $[a, b]$ with $k:(0, \infty) \rightarrow(0, \infty)$, $k(t)=\frac{1}{t}$ and $g(t)=\ln t, t \in I$. By making use of Corollary 4 we get

$$
\begin{gathered}
\frac{1}{2 h\left(\frac{1}{2}\right)} \max \left\{\frac{1-\lambda}{h(1-\lambda)}, \frac{\lambda}{h(\lambda)}\right\} f^{-1}(\sqrt{x y}) \\
\geq \frac{1}{2 h\left(\frac{1}{2}\right)}\left\{\lambda f^{-1}\left(x^{\frac{2-\lambda}{2}} y^{\frac{\lambda}{2}}\right)+(1-\lambda) f^{-1}\left(x^{\frac{1-\lambda}{2}} y^{\frac{\lambda+1}{2}}\right)\right\} \\
\geq \frac{1}{\ln \left(\frac{y}{x}\right)} \int_{x}^{y} \frac{f^{-1}(t)}{t} d t \\
\geq\left[f^{-1}\left(x^{1-\lambda} y^{\lambda}\right)+\lambda f^{-1}(x)+(1-\lambda) f^{-1}(y)\right] \int_{0}^{1} h(t) d t \\
\geq\left\{[h(1-\lambda)+\lambda] f^{-1}(x)+[h(\lambda)+1-\lambda] f^{-1}(y)\right\} \int_{0}^{1} h(t) d t,
\end{gathered}
$$

for any $\lambda \in[0,1]$ and for $y, x \in[a, b]$ with $y \neq x$.

\section{Applications for $H A, H G$ and $H H$-h-Convex Functions}

Let $f:[a, b] \subset(0, \infty) \rightarrow \mathbb{R}$ be an $H A$-h-convex function on the interval $[a, b]$. This is equivalent to the fact that $f$ is composite- $g^{-1} h$-convex on $[a, b]$ with the increasing function $g(t)=-\frac{1}{t}$. Then by applying Corollary 4 for $k(t)=t$, we have the inequalities

$$
\begin{aligned}
& \frac{1}{2 h\left(\frac{1}{2}\right)} \min \left\{\frac{1-\lambda}{h(1-\lambda)}, \frac{\lambda}{h(\lambda)}\right\} f\left(\frac{2 x y}{x+y}\right) \\
& \leq \frac{1}{2 h\left(\frac{1}{2}\right)}\left\{(1-\lambda) f\left(\frac{2 x y}{(1-\lambda) x+(\lambda+1) y}\right)+\lambda f\left(\frac{2 x y}{(2-\lambda) x+\lambda y}\right)\right\} \\
& \leq \frac{x y}{y-x} \int_{x}^{y} \frac{f(t)}{t^{2}} d t \\
& \leq \frac{1}{2}\left[f\left(\frac{x y}{(1-\lambda) x+\lambda y}\right)+(1-\lambda) f(x)+\lambda f(y)\right] \int_{0}^{1} h(t) d t \\
& \quad \leq\{[h(1-\lambda)+\lambda] f(x)+[h(\lambda)+1-\lambda] f(y)\} \int_{0}^{1} h(t) d t
\end{aligned}
$$

for any $\lambda \in[0,1]$ and for $y, x \in[a, b]$ with $y \neq x$.

Let $f:[a, b] \subset(0, \infty) \rightarrow(0, \infty)$ be an $H G$-h-convex function on the interval $[a, b]$. This is equivalent to the fact that $f$ is $k$-composite- $g^{-1} h$-convex on $[a, b]$ 
with $k:(0, \infty) \rightarrow \mathbb{R}, k(t)=\ln t$ and $g(t)=-\frac{1}{t}, t \in[a, b]$. Then by applying Corollary 4 , we have the inequalities

$$
\begin{aligned}
& {\left[f\left(\frac{2 x y}{x+y}\right)\right]^{\frac{1}{2 h\left(\frac{1}{2}\right)} \min \left\{\frac{1-\lambda}{h(1-\lambda)}, \frac{\lambda}{h(\lambda)}\right\}}} \\
& \leq\left[f^{1-\lambda}\left(\frac{2 x y}{(1-\lambda) x+(\lambda+1) y}\right) f^{\lambda}\left(\frac{2 x y}{(2-\lambda) x+\lambda y}\right)\right]^{\frac{1}{2 h\left(\frac{1}{2}\right)}} \\
& \leq \exp \left(\frac{x y}{y-x} \int_{x}^{y} \frac{\ln f(t)}{t^{2}} d t\right) \\
& \leq\left[f\left(\frac{x y}{(1-\lambda) x+\lambda y}\right)[f(x)]^{1-\lambda}[f(y)]^{\lambda}\right]^{\int_{0}^{1} h(t) d} \\
& \leq\left\{f^{[h(1-\lambda)+\lambda]}(x) f^{[h(\lambda)+1-\lambda]}(y)\right\}^{\int_{0}^{1} h(t) d t}
\end{aligned}
$$

for any $\lambda \in[0,1]$ and for $y, x \in[a, b]$ with $y \neq x$.

Let $f:[a, b] \subset(0, \infty) \rightarrow(0, \infty)$ be an $H H$ - $h$-convex function on the interval $[a, b]$. This is equivalent to the fact that $f$ is $k$-composite- $g^{-1} h$-concave on $[a, b]$ with $k:(0, \infty) \rightarrow(0, \infty), k(t)=\frac{1}{t}$ and $g(t)=-\frac{1}{t}, t \in[a, b]$. Then by applying Corollary 4 , we have the inequalities

$$
\begin{aligned}
& \frac{1}{2 h\left(\frac{1}{2}\right)} \max \left\{\frac{1-\lambda}{h(1-\lambda)}, \frac{\lambda}{h(\lambda)}\right\} f^{-1}\left(\frac{2 x y}{x+y}\right) \\
\geq & \frac{1}{2 h\left(\frac{1}{2}\right)}\left\{\lambda f^{-1}\left(\frac{2 x y}{(2-\lambda) x+\lambda y}\right)+(1-\lambda) f^{-1}\left(\frac{2 x y}{(1-\lambda) x+(\lambda+1) y}\right)\right\} \\
\geq & \frac{x y}{y-x} \int_{x}^{y} \frac{f^{-1}(t)}{t^{2}} d t \\
\geq & {\left[f^{-1}\left(\frac{x y}{(1-\lambda) x+\lambda y}\right)+\lambda f^{-1}(x)+(1-\lambda) f^{-1}(y)\right] \int_{0}^{1} h(t) d t } \\
& \geq\left\{[h(1-\lambda)+\lambda] f^{-1}(x)+[h(\lambda)+1-\lambda] f^{-1}(y)\right\} \int_{0}^{1} h(t) d t,
\end{aligned}
$$

for $y, x \in[a, b]$ with $y \neq x$.

Applications for $p, r$-convex and $\log E x p$ convex functions can also be provided. However the details are not presented here.

\section{REFERENCES}

[1] M. W. Alomari, Some properties of $h-M N$-Convexity and Jensen's type inequalities. Preprints 2017, 2017100176 (doi: 10.20944/preprints201710.0176.v1).

[2] M. W. Alomari and M. Darus, The Hadamard's inequality for s-convex function. Int. J. Math. Anal. (Ruse) 2 (2008), no. 13-16, 639-646.

[3] M. W. Alomari and M. Darus, Hadamard-type inequalities for s-convex functions. Int. Math. Forum 3 (2008), no. 37-40, 1965-1975.

[4] G. A. Anastassiou, Univariate Ostrowski inequalities, revisited. Monatsh. Math., 135 (2002), no. $3,175-189$.

[5] G. D. Anderson, M. K. Vamanamurthy and M. Vuorinen, Generalized convexity and inequalities, J. Math. Anal. Appl. 335 (2007) 1294-1308. 
[6] N. S. Barnett, P. Cerone and S. S. Dragomir, Some new inequalities for HermiteHadamard divergence in information theory, in Stochastic Analysis \& Applications, Vol. 3, Eds. Y. J. Cho, J. K. Kim and Y. K. Choi, pp. 7-19, Nova Sci. Publishers, 2003, ISBN 1-59033-860-X. Preprint RGMIA Res. Rep. Coll. 5 (2002), Issue 4, Art. 8. [Online http://rgmia.org/papers/v5n4/NIHHDIT.pdf] .

[7] N. S. Barnett, P. Cerone, S. S. Dragomir, M. R. Pinheiro,and A. Sofo, Ostrowski type inequalities for functions whose modulus of the derivatives are convex and applications. Inequality Theory and Applications, Vol. 2 (Chinju/Masan, 2001), 19-32, Nova Sci. Publ., Hauppauge, NY, 2003. Preprint: RGMIA Res. Rep. Coll. 5 (2002), No. 2, Art. 1 [Online http://rgmia.org/papers/v5n2/Paperwapp2q.pdf].

[8] E. F. Beckenbach, Convex functions, Bull. Amer. Math. Soc. 54(1948), 439-460.

[9] M. Bombardelli and S. Varošanec, Properties of h-convex functions related to the HermiteHadamard-Fejér inequalities. Comput. Math. Appl. 58 (2009), no. 9, 1869-1877.

[10] W. W. Breckner, Stetigkeitsaussagen für eine Klasse verallgemeinerter konvexer Funktionen in topologischen linearen Räumen. (German) Publ. Inst. Math. (Beograd) (N.S.) 23(37) (1978), 13-20.

[11] W. W. Breckner and G. Orbán, Continuity properties of rationally s-convex mappings with values in an ordered topological linear space. Universitatea "Babeş-Bolyai", Facultatea de Matematica, Cluj-Napoca, 1978. viii+92 pp.

[12] P. Cerone and S. S. Dragomir, Midpoint-type rules from an inequalities point of view, Ed. G. A. Anastassiou, Handbook of Analytic-Computational Methods in Applied Mathematics, CRC Press, New York. 135-200.

[13] P. Cerone and S. S. Dragomir, New bounds for the three-point rule involving the RiemannStieltjes integrals, in Advances in Statistics Combinatorics and Related Areas, C. Gulati, et al. (Eds.), World Science Publishing, 2002, 53-62.

[14] P. Cerone, S. S. Dragomir and J. Roumeliotis, Some Ostrowski type inequalities for $n$-time differentiable mappings and applications, Demonstratio Mathematica, 32(2) (1999), 697712 .

[15] G. Cristescu, Hadamard type inequalities for convolution of $h$-convex functions. Ann. Tiberiu Popoviciu Semin. Funct. Equ. Approx. Convexity 8 (2010), 3-11.

[16] S. S. Dragomir, Ostrowski's inequality for monotonous mappings and applications, J. KSIAM, 3(1) (1999), 127-135.

[17] S. S. Dragomir, The Ostrowski's integral inequality for Lipschitzian mappings and applications, Comp. Math. Appl., 38 (1999), 33-37.

[18] S. S. Dragomir, On the Ostrowski's inequality for Riemann-Stieltjes integral, Korean J. Appl. Math., 7 (2000), 477-485.

[19] S. S. Dragomir, On the Ostrowski's inequality for mappings of bounded variation and applications, Math. Ineq. 83 Appl., 4(1) (2001), 33-40.

[20] S. S. Dragomir, On the Ostrowski inequality for Riemann-Stieltjes integral $\int_{a}^{b} f(t) d u(t)$ where $f$ is of Hölder type and $u$ is of bounded variation and applications, J. KSIAM, 5(1) (2001), 35-45.

[21] S. S. Dragomir, Ostrowski type inequalities for isotonic linear functionals, J. Inequal. Pure \& Appl. Math., 3(5) (2002), Art. 68.

[22] S. S. Dragomir, An inequality improving the first Hermite-Hadamard inequality for convex functions defined on linear spaces and applications for semi-inner products. J. Inequal. Pure Appl. Math. 3 (2002), no. 2, Article 31, 8 pp.

[23] S. S. Dragomir, An inequality improving the first Hermite-Hadamard inequality for convex functions defined on linear spaces and applications for semi-inner products, J. Inequal. Pure Appl. Math. 3 (2002), No. 2, Article 31.

[24] S. S. Dragomir, An inequality improving the second Hermite-Hadamard inequality for convex functions defined on linear spaces and applications for semi-inner products, J. Inequal. Pure Appl. Math. 3 (2002), No.3, Article 35.

[25] S. S. Dragomir, An Ostrowski like inequality for convex functions and applications, Revista Math. Complutense, 16(2) (2003), 373-382.

[26] S. S. Dragomir, Operator Inequalities of Ostrowski and Trapezoidal Type. Springer Briefs in Mathematics. Springer, New York, 2012. x+112 pp. ISBN: 978-1-4614-1778-1 
[27] S. S. Dragomir, Ostrowski type inequalities for Lebesgue integral: a survey of recent results. Aust. J. Math. Anal. Appl. 14 (2017), no. 1, Art. 1, 283 pp. [Online http://ajmaa.org/cgi-bin/paper.pl?string=v14n1/V14I1P1.tex] .

[28] S. S. Dragomir, Inequalities for a generalized finite Hilbert transform of convex functions, Preprint RGMIA Res. Rep. Coll. 21 (2018), Art.

[29] S. S. Dragomir and C. E. M. Pearce, Selected Topics on HermiteHadamard Inequalities and Applications, RGMIA Monographs, 2000. [Online http://rgmia.org/monographs/hermite_hadamard.html] .

[30] S. S. Dragomir, New inequalities of Hermite-Hadamard type for log-convex functions. Khayyam J. Math. 3 (2017), no. 2, 98-115.

[31] Dragomir, S. S. Inequalities of Hermite-Hadamard type for $h$-convex functions on linear spaces. Proyecciones 34 (2015), no. 4, 323-341.

[32] S. S. Dragomir, Inequalities of Hermite-Hadamard type for $G A$-convex functions, to appear in Annales Mathematicae Silesianae, Preprint RGMIA Res. Rep. Coll. 18 (2015), Art. 30. [Online http://rgmia.org/papers/v18/v18a30.pdf].

[33] S. S. Dragomir, Inequalities of Hermite-Hadamard type for GG-convex functions, Preprint RGMIA, Research Report Collection, 18 (2015), Art. 71, 15 pp., [Online http://rgmia.org/papers/v18/v18a71.pdf] .

[34] S. S. Dragomir, Some integral inequalities of Hermite-Hadamard type for $G G$-convex functions, Mathematica (Cluj), 59 (82), No 1-2, 2017, pp. 47-64. Preprint RGMIA, Research Report Collection, 18 (2015), Art. 74. [Online http://rgmia.org/papers/v18/v18a74.pdf] .

[35] S. S. Dragomir, Inequalities of Hermite-Hadamard type for $H A$-convex functions, Maroccan J. Pure 83 Appl. Analysis, Volume 3 (1), 2017, Pages 83-101. Preprint, RGMIA Res. Rep. Coll. 18 (2015), Art. 38. [Online http://rgmia.org/papers/v18/v18a38.pdf] .

[36] S. S. Dragomir, Inequalities of Hermite-Hadamard type for $H G$-convex functions, Probl. Anal. Issues Anal. Vol. 6 (24), No. 2, 2017 1-17. Preprint, RGMIA Res. Rep. Coll. 18 (2015), Art. 79. [Online http://rgmia.org/papers/v18/v18a79.pdf].

[37] S. S. Dragomir, Inequalities of Hermite-Hadamard type for $H H$-convex functions, to appear in Acta et Commentationes Universitatis Tartuensis de Mathematica, Preprint, RGMIA Res. Rep. Coll. 18 (2015), Art. 80. [Online http://rgmia.org/papers/v18/v18a80.pdf] .

[38] S. S. Dragomir, Inequalities of Hermite-Hadamard type for composite convex functions, Preprint, RGMIA Res. Rep. Coll. 21 (2018), Art.

[39] S. S. Dragomir, P. Cerone, J. Roumeliotis and S. Wang, A weighted version of Ostrowski inequality for mappings of Hölder type and applications in numerical analysis, Bull. Math. Soc. Sci. Math. Romanie, 42(90) (4) (1999), 301-314.

[40] S.S. Dragomir and S. Fitzpatrick, The Hadamard inequalities for s-convex functions in the second sense. Demonstratio Math. 32 (1999), no. 4, 687-696.

[41] S.S. Dragomir and S. Fitzpatrick,The Jensen inequality for s-Breckner convex functions in linear spaces. Demonstratio Math. 33 (2000), no. 1, 43-49.

[42] S. S. Dragomir and B. Mond, On Hadamard's inequality for a class of functions of Godunova and Levin. Indian J. Math. 39 (1997), no. 1, 1-9.

[43] S. S. Dragomir and C. E. M. Pearce, On Jensen's inequality for a class of functions of Godunova and Levin. Period. Math. Hungar. 33 (1996), no. 2, 93-100.

[44] S. S. Dragomir and C. E. M. Pearce, Quasi-convex functions and Hadamard's inequality, Bull. Austral. Math. Soc. 57 (1998), 377-385.

[45] S. S. Dragomir, J. Pečarić and L. Persson, Some inequalities of Hadamard type. Soochow J. Math. 21 (1995), no. 3, 335-341.

[46] S. S. Dragomir and Th. M. Rassias (Eds), Ostrowski Type Inequalities and Applications in Numerical Integration, Kluwer Academic Publisher, 2002.

[47] S. S. Dragomir and S. Wang, A new inequality of Ostrowski's type in $L_{1}$-norm and applications to some special means and to some numerical quadrature rules, Tamkang J. of Math., 28 (1997), 239-244.

[48] S. S. Dragomir and S. Wang, Applications of Ostrowski's inequality to the estimation of error bounds for some special means and some numerical quadrature rules, Appl. Math. Lett., 11 (1998), 105-109.

[49] S. S. Dragomir and S. Wang, A new inequality of Ostrowski's type in $L_{p}$-norm and applications to some special means and to some numerical quadrature rules, Indian J. of Math., 40(3) (1998), 245-304. 
[50] A. El Farissi, Simple proof and refinement of Hermite-Hadamard inequality, J. Math. Ineq. 4 (2010), No. 3, 365-369.

[51] L. Fejér, Über die Fourierreihen, II, (In Hungarian) Math. Naturwiss, Anz. Ungar. Akad. Wiss., 24 (1906), 369-390.

[52] E. K. Godunova and V. I. Levin, Inequalities for functions of a broad class that contains convex, monotone and some other forms of functions. (Russian) Numerical mathematics and mathematical physics (Russian), 138-142, 166, Moskov. Gos. Ped. Inst., Moscow, 1985

[53] H. Hudzik and L. Maligranda, Some remarks on s-convex functions. Aequationes Math. 48 (1994), no. 1, 100-111.

[54] E. Kikianty and S. S. Dragomir, Hermite-Hadamard's inequality and the p-HH-norm on the Cartesian product of two copies of a normed space, Math. Inequal. Appl. (in press)

[55] U. S. Kirmaci, M. Klaričić Bakula, M. E Özdemir and J. Pečarić, Hadamard-type inequalities for s-convex functions. Appl. Math. Comput. 193 (2007), no. 1, 26-35.

[56] M. A. Latif, On some inequalities for h-convex functions. Int. J. Math. Anal. (Ruse) 4 (2010), no. $29-32,1473-1482$.

[57] D. S. Mitrinović and I. B. Lacković, Hermite and convexity, Aequationes Math. 28 (1985), 229-232.

[58] D. S. Mitrinović and J. E. Pečarić, Note on a class of functions of Godunova and Levin. $C$. R. Math. Rep. Acad. Sci. Canada 12 (1990), no. 1, 33-36.

[59] P. Montel, Sur les functions convexes et les fonctions sousharmoniques, Journal de Math., 9 (1928), 7, 29-60.

[60] C. P. Niculescu, Convexity according to the geometric mean, Math. Inequal. Appl., 3, (2000), 2, $155-167$.

[61] C. E. M. Pearce, J. Pečarić and V. Šimić, Stolarsky means and Hadamard's inequality. J. Math. Anal. Appl. 220, 99-109 (1998)

[62] C. E. M. Pearce and A. M. Rubinov, P-functions, quasi-convex functions, and Hadamard-type inequalities. J. Math. Anal. Appl. 240 (1999), no. 1, 92-104.

[63] J. E. Pečarić and S. S. Dragomir, On an inequality of Godunova-Levin and some refinements of Jensen integral inequality. Itinerant Seminar on Functional Equations, Approximation and Convexity (Cluj-Napoca, 1989), 263-268, Preprint, 89-6, Univ. "Babeş-Bolyai", Cluj-Napoca, 1989.

[64] J. Pečarić and S. S. Dragomir, A generalization of Hadamard's inequality for isotonic linear functionals, Radovi Mat. (Sarajevo) 7 (1991), 103-107.

[65] M. Radulescu, S. Radulescu and P. Alexandrescu, On the Godunova-Levin-Schur class of functions. Math. Inequal. Appl. 12 (2009), no. 4, 853-862.

[66] M. Z. Sarikaya, A. Saglam, and H. Yildirim, On some Hadamard-type inequalities for hconvex functions. J. Math. Inequal. 2 (2008), no. 3, 335-341.

[67] E. Set, M. E. Özdemir and M. Z. Sarıkaya, New inequalities of Ostrowski's type for s-convex functions in the second sense with applications. Facta Univ. Ser. Math. Inform. 27 (2012), no. 1, 67-82.

[68] M. Z. Sarikaya, E. Set and M. E. Özdemir, On some new inequalities of Hadamard type involving h-convex functions. Acta Math. Univ. Comenian. (N.S.) 79 (2010), no. 2, 265-272.

[69] M. Tunç, Ostrowski-type inequalities via h-convex functions with applications to special means. J. Inequal. Appl. 2013, 2013:326.

[70] S. Varošanec, On h-convexity. J. Math. Anal. Appl. 326 (2007), no. 1, 303-311.

[71] K. S. Zhang and J. P. Wan, p-convex functions and their properties. Pure Appl. Math. 23(1), 130-133 (2007).

${ }^{1}$ Mathematics, College of Engineering \& Science, Victoria University, PO Box 14428 , Melbourne City, MC 8001, Australia.

E-mail address: sever.dragomir@vu.edu.au

$U R L:$ http://rgmia.org/dragomir

${ }^{2}$ DSt-nRF Centre of Excellence in the Mathematical, and Statistical Sciences, School of Computer Science, \& Applied Mathematics, University of the WitwaterSrand,, Private Bag 3, Johannesburg 2050, South Africa 